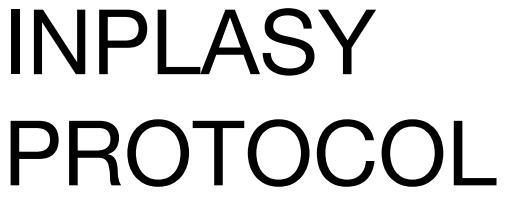

To cite: Ma et al. Effect of dexmedetomidine for the treatment of long-term mechanical ventilation: $A$ systematic review and metaanalysis of randomized controlled trials. Inplasy protocol 202080085. doi: 10.37766/inplasy2020.8.0085

Received: 19 August 2020

Published: 20 August 2020

Corresponding author: Wang Jing

13573195707@163.com

Author Affiliation: Department of Critical Care Medicine, Qilu Hospital, Shandong University

Support: None.

Review Stage at time of this submission: The review has not yet started.

\section{Effect of dexmedetomidine for the treatment of long-term mechanical ventilation: A systematic review and meta-analysis of randomized controlled trials}

\author{
Ma, S1; Guo, L2; Zhang, Y3; Wang, J4.
}

Review question / Objective: The primary aim of this review was to examine whether dexmedetomidine reduces the time of mechanical vetilation in intensive care patients.

Condition being studied: Sedation in intensive care patients has been shown to reduce discomfort associated with nursing interventions, increase tolerance to mechanical ventilation, prevent accidental removal of devices, and reduce metabolic requirements during cardiovascular and respiratory instability. Dexmedetomidine, a highly selective a2-adrenoreceptor agonist, has increasingly used for sedation in ICU. Dexmedetomidine was more effective than midazolam and propofol in early goal-directed sedation. Studies have demonstrated that dexmedetomidine can shorten the time of mechanical ventilation in ICU patients. However, some other relevant studies have different conclusions. There is no clear conclusion on the effect of dexmedetomidine, especially for patients with mechanical ventilation over 24 hours.

INPLASY registration number: This protocol was registered with the International Platform of Registered Systematic Review and Meta-Analysis Protocols (INPLASY) on 20 August 2020 and was last updated on 20 August 2020 (registration number INPLASY202080085).

Conflicts of interest:

None.

\section{INTRODUCTION}

Review question / Objective: The primary aim of this review was to examine whether dexmedetomidine reduces the time of mechanical vetilation in intensive care patients.

Rationale: We will search studies in the database, the results were screened according to the criteria, then we include the literature, extracte data, and finally conduct the systematic review and metaanalysis.

Condition being studied: Sedation in intensive care patients has been shown to reduce discomfort associated with nursing interventions, increase tolerance to mechanical ventilation, prevent accidental removal of devices, and reduce metabolic requirements during cardiovascular and respiratory instability. Dexmedetomidine, a highly selective a2-adrenoreceptor agonist, 
has increasingly used for sedation in ICU. Dexmedetomidine was more effective than midazolam and propofol in early goaldirected sedation. Studies have demonstrated that dexmedetomidine can shorten the time of mechanical ventilation in ICU patients. However, some other relevant studies have different conclusions. There is no clear conclusion on the effect of dexmedetomidine, especially for patients with mechanical ventilation over 24 hours.

\section{METHODS}

Search strategy: We will search the following databases: the Cochrane Central Register of Controlled Trials, PubMed, Embase, Web of Science from inception to 30 September 2020. The strategies of searching will be developed using Medical Subject Heading terms, such as 'dexmedetomidine' ,'ICU','mechanical vetilation' and corresponding text words and no language restrictions will be imposed.

Participant or population: Adult patients (aged 18 years or older) with mechanical ventilation Patients with lung surgery or physical lung injury will be excluded.

Intervention: Sedation only with dexmedetomidine.

Comparator: Treated with other sedative agents.

Study designs to be included: Randomized controlled trials(RCTs).

Eligibility criteria: Study designs: this review will include only RCTs. Patients: adult patients with mechanical ventilation(aged 18 years or older), mechanically ventilated more than $24 \mathrm{~h}$ is not less than $24 \mathrm{~h}$, Patients with lung surgery or physical lung injury will be excluded. Interventions: a comparison between dexmedetomidine and placebo or other sedative agents (including benzodiazepines, propofol and other sedatives) will be included.
Information sources: We will search the following databases: the Cochrane Central Register of Controlled Trials, PubMed, Embase, Web of Science. When a study either overlapped or was a duplicate of another study, we will contact the study authors for clarification and, if confirmed, will use the publication with the more detailed data for this meta-analysis and combined the additional data.

Main outcome(s): Time of mechanical ventilation after ICU admission.

Additional outcome(s): 1. All-cause mortality within 28 days after ICU admission; if mortality was assessed at other follow-up times (eg, ICU mortality) or the follow-up times was not reported, we arbitrarily consider them as 28 day mortality and conduct subgroup analysis. 2. Length of ICU stay. 3. Incidence of adverse events.

Data management: Two reviewers independently will extract data from the articles using a predesigned data extraction form, and any disagreement will be resolved by consulting a third reviewer. When a study either overlapped or was a duplicate of another study, we will contact the study authors for clarification and, if confirmed, will use the publication with the more detailed data for this meta-analysis and combined the additional data. The following information will be extracted: Basic information: title, publication year, name of authors, centres (single-centre, multi-centre), registration identification, funding. Patients: sample size, country/ countries, mean age, diseases, target sedation range. Interventions: administration of drugs, treatment duration. Comparator: administration of placebo, treatment duration. Outcomes: Time of mechanical ventilation after ICU admission, all-cause mortality within 28 days after ICU admission, length of ICU stay, incidence of adverse events, such as hypotension and bradycardia.

Quality assessment / Risk of bias analysis: The methodological quality of the included studies will be assessed using the 
Cochrane Bias risk tool. The Cochrane bias risk tool consists of seven areas, including sequence generation, allocation concealment, participant and person blindness, outcome assessment blindness, incomplete outcome data, selective reporting, and other biases. Each area is classified as "low," "high," or "unclear" bias risk. Methodological quality assessments will be conducted independently by two reviewers, and objections will be resolved by discussion or by a third reviewer.

Strategy of data synthesis: The metaanalysis will use Stata/SE 15.0. For the results of this systematic review, dichotomous data and continuous data will be calculated in the form of risk ratios and standardized mean differences, respectively, with corresponding $\mathrm{Cl}$ of $95 \%$. Statistical heterogeneity between studies was assessed by tests (statistically significant at $P<0.05$ ) and quantified by $\mathbf{I}$ statistics $(25 \%, 50 \%$, and $75 \%$ were considered low, medium, and high heterogeneity, respectively). Randomeffects model were used to aggregate data on the assumption that individual RCTS differed in patient populations, depth of sedation, trial grouping and dose of dexmedetomidine or other sedatives. When significant heterogeneity is found, we will explore possible explanations in the subgroup analysis. If there is insufficient data available (less than two studies) for comparison, we use narrative, qualitative descriptions rather than meta-analyses. Continuous data that presented as medians and IQR or ranges will be converted to means and SD as the instruction of Cochrane Handbook. The median value and SD will be estimated based on the number of samples, IQR and ranges.we will exclude these estimates and conduct a sensitivity analysis to assess the robustness of the results.

Subgroup analysis: If sufficient data are available, we will perform a subgroup analysis of the main results as follows. Mechanical ventilation time: 1. Types of patients: medical patients, surgical patients, sepsis patients, and others. 2.
Target sedation range: deep sedation or mild sedation. 3. Control group: propofol, midazolam, etc. 4. Drug injection speed: high or low injection speed. 5. Other factors of control group or experimental group: nursing standard, other factors.

Sensibility analysis: To assess whether the results of the system evaluation are robust, the main results of the sensitivity analysis we will perform include studies of small sample sizes, methods of low methodological quality (unclear reporting blinding and allocation concealment), and studies of high percentage withdrawals (more than 10\%). We will conduct a sensitivity analysis of the continuous results to exclude studies reporting data other than mean deviation and standard deviation.

\section{Language: No limitation.}

Country(ies) involved: The review will be conducted in China. Countries be involved will be list as basic information in the study.

Keywords: dexmedetomidine, mechanical ventilation, sedation.

\section{Contributions of each author:}

Author 1 - Shuo Ma - This author will scan the titles and abstracts of the searched literatures independently and in duplicate against the inclusion criteria, and extract data from the articles using a predesigned data extraction form.Author 1 will draft the manuscript.

Author 2 - Lianliang Guo - This author will extract data from the articles using a predesigned data extraction form. And develop a search strategy.

Author 3 - Yingying Zhang - This author will scan the titles and abstracts of the searched literatures independently and in duplicate against the inclusion criteria.And made a data extraction table.

Author 4 - Jing Wang - This author will complete data analysis and save the graphs involved. Author 4 read, provided feedback and approved the final manuscript. 